\title{
Speed of sound in outer planet atmospheres
}

\author{
Ralph D. Lorenz* \\ Lunar and Planetary Laboratory, University of Arizona, Tucson AZ 85721-0092, U.S.A.
}

Received 3 March 1998; received in revised form 4 August 1998; accepted 6 September 1998

\begin{abstract}
I compute speed of sound profiles for the four giant planets using simple atmosphere models, and explore the effect on the profiles of ortho: para hydrogen conversion, and the drop in mixing ratio of condensible species at the tropopause. The speed of sound is in general somewhat lower (principally due to lower temperature and higher relative molecular mass) in Uranus and Neptune than in Saturn and Jupiter. Furthermore, the two outer planets show dramatic $(\sim 10 \%)$ changes in sound speed due to the condensation of methane, suggesting that sound speed measurement on a probe is a useful diagnostic of vertical structure. Sound Speed measurements for Jupiter and Saturn need to be made with much higher accuracy ( $\sim 0.02 \%$ for Jupiter and $0.1 \%$ for Saturn) to usefully constrain structure and transport. Some instrumental considerations are discussed. (C) 1999 Elsevier Science Ltd. All rights reserved.
\end{abstract}

\section{Introduction}

The speed of sound is a common diagnostic for temperature in terrestrial science, and has been long proposed for measurement in planetary atmospheres, as a diagnostic for composition, temperature and hydrogen transport. In this paper I review the concepts and instruments to date, and investigate by means of model atmospheres what speed of sound measurements might tell us about the atmospheres of the outer planets, and how accurately the speed of sound and supporting measurements have to be made in order to usefully constrain models.

\section{Review}

Terrestrial measurements of the speed of sound are employed in five notable areas. First is to measure air temperature in micrometeorological applications - a significant advantage is that the temperature of the air is sensed directly enabling measurements over very short temporal and spatial scales, not limited by the thermal time constant of a sensor. Second, acoustic thermometry is widely employed in industrial applications, such as to measure the temperature of flue gasses from burners; the high temperature and particulate loading of these gas streams poses a significant reliability and lifetime penalty on sensors placed in them. A review of the technique

* Tel.: + 1520621 5585; fax: +1 520621 4933; e-mail: rlorenz@lpl. arizona.edu ('acoustic pyrometry') is given by Kleppe (1996). Third, the transit time of an acoustic wave across wide spans of ocean provides information on the ocean temperature over vast ( $>1000 \mathrm{~km}$ wide) regions that would otherwise be difficult to reach, (e.g., Spiesberger and Tappert, 1996). Fourth, the speed of sound is an easy laboratory parameter to measure, and can be used to determine thermodynamic properties and equations of state (e.g., Estrada-Alexanders et al., 1994). Finally, SODAR (Sound Detection And Ranging) methods are used in boundary layer research: sound pulses are transmitted upwards from the ground and echoes from clouds and/or temperature discontinuities sensed by their echo. The reflection of sound from clear-air inhomogeneities was noted by Tyndall over the English channel (his 1896 book is still a very readable and worthwhile reference) - useful modern reviews are given by Neff and Coulter (1986) and Weill and Lehmann (1990).

In these applications, the composition of the medium (and hence its relative molecular mass and specific heat) is either known or assumed, so that the sound speed relates directly to temperature. For planetary applications, although temperature can be constrained by the measurement of sound speed, it is generally more useful to determine temperature separately and thence constrain the medium properties.

Acoustic measurements for spacecraft were proposed as early as 1966 (Hanel and Strange, 1966), before in-situ planetary measurements of any kind had been made. Their concept was to measure the sound speed in a temperature-controlled spiral duct, thus constraining the relative molecular mass and specific heat. The application 
in mind was to resolve the relative abundances of $\mathrm{CO}_{2}$, $\mathrm{N}_{2}$ and $\mathrm{Ar}$ in the then-unknown Martian atmosphere.

Acoustic sensors have been flown on Soviet Venus landers, although with the intent of detecting acoustic emission due to thunder. Although events were detected (Ksanfomality et al., 1983a) it is unclear whether these were indeed due to thunder, or merely aeroacoustic noise caused by the turbulent airflow over the probe (both sources peak at frequencies of a few Hertz). Data from these sensors was used, on the surface, to constrain the surface windspeeds (Ksanfomality et al., 1983b).

More recently, the first outer planet acoustic measurement has been sent on its way. The Surface Science Package (SSP) on the ESA Huygens probe to Titan includes (Zarnecki et al., 1997) an acoustic sounder. This device, acting as a SODAR (Sonic Detection and Ranging) will measure topographic roughness at the landing site prior to impact, in the same way as a radar altimeter. Additionally, it will also constrain the depth of any liquid hydrocarbon deposit the probe might land in by sensing an echo from the bottom, and possibly detect acoustic backscatter from raindrops during the descent. The package also includes a speed of sound instrument, to constrain the composition of surface liquid and facilitate the measurement of lake depth. This sensor will also operate in the atmosphere, sampling every $10 \mathrm{~m}$ altitude or so-a better altitude resolution than the temperature sensors of the Huygens Atmospheric Structure Instrument ('HASI', Fulchignoni et al., 1997). The combination of the HASI temperature measurement and the SSP speed of sound may provide useful information on the altitude variation of the methane mixing ratio (via its effect on the relative molecular mass), which varies from around $8 \%$ near the surface to about $2 \%$ at the tropopause, e.g., Yelle et al. (1997).

In this paper, I consider what might be learned from a speed of sound measurement in the outer planets. The present NASA Planetary Exploration Roadmap includes plans for a Jupiter multiprobe mission and a Neptune orbiter (which might include an atmosphere probe). Additionally, now that Cassini is safely on its way and could conceivably provide a relay capability for a fasttrack Discovery-class Saturn probe mission in the latter half of the next decade, it is appropriate to consider that body too. Uranus is considered also, for completeness. I defer discussion of Titan, from which sound speed measurements may be expected in 2004, to a future paper.

\section{Speed of sound-a diagnostic of composition and transport}

The sound speed in an ideal gas may be given by (e.g., Rogers and Mayhew 1967; Hanel and Strange, 1966)

$c=\sqrt{\gamma T R_{0} / M}$ where $\gamma$ is the ratio of specific heats, $T$ is the temperature in Kelvin, $R_{0}$ is the universal gas constant $\left(8314 \mathrm{~J} \mathrm{~K}^{-1}\right.$ $\left.\mathrm{mol}^{-1}\right) . M$ is the mean relative molecular mass (RMM) of the gas mixture, the sum of the RMMs of the component gasses $M_{\mathrm{i}}$, weighted by their mole fraction $X_{\mathrm{i}}$.

Note that the ratio of specific heats is weighted by the mass fractions of the components, rather than their mixing ratio. As discussed in Hanel and Strange (1966), this makes the lines of varying composition on a plot of $\gamma$ vs $M$ curved, rather than straight.

On Titan, where the nitrogen-dominated atmosphere approaches its condensation temperature and compressibility effects become significant (e.g., Lindal et al., 1983), the ideal gas formula may not be strictly applicable. However, in the atmosphere of the outer planets, dominated by hydrogen and helium, the ideal gas law is sufficiently accurate for the regions of interest, despite the cold temperatures. Chapter 5 of Lewis (1995) discusses nonidealities at great depths in the Jovian planets, but these are at too high pressure and temperatures to be relevant for measurement by spacecraft, the subject of the present paper.

An additional factor, beyond the molecular composition, is the spin state of hydrogen molecules (See, e.g., Massie and Hunten, 1982, and Appendix VIII of Chamberlain and Hunten, 1987). The two spin states, ortho and para, have significantly different specific heats at constant pressure and hence ratios of specific heats: see Fig. 1. The two states have an equilibrium ratio that depends on temperature, see Fig. 2. In the high temperature limit, the fraction of hydrogen $f_{\mathrm{p}}$ in the para form is $25 \%$; at temperatures below $100 \mathrm{~K}$ (and thus relevant to the tropopause regions in the outer planet atmospheres), the para fraction rises above $50 \%$. Because hydrogen is the dominant constituent, this otherwise obscure physical effect assumes considerable importance, and can modify the lapse rate measurably (Massie and Hunten, 1982), and substantially influence convective processes (Conrath and Gierasch, 1984; Smith and Gierasch, 1995).

\section{Model atmospheres and speeds of sound}

Model atmospheres for the four outer planets have been constructed to determine speed of sound profiles. These models are not especially rigorous, and include only one condensible constituent for simplicity, but do serve to show the different regimes in the four planets, and how the sound speed is related to the ortho:para hydrogen and the mixing ratio profile of the condensible species.

Data for the pressure:temperature profiles was obtained from Seiff et al. (1997), Ingersoll et al. (1984), Conrath et al. (1991) and Gautier et al. (1995), for Jupiter, Saturn, Uranus and Neptune, respectively. A point in the 


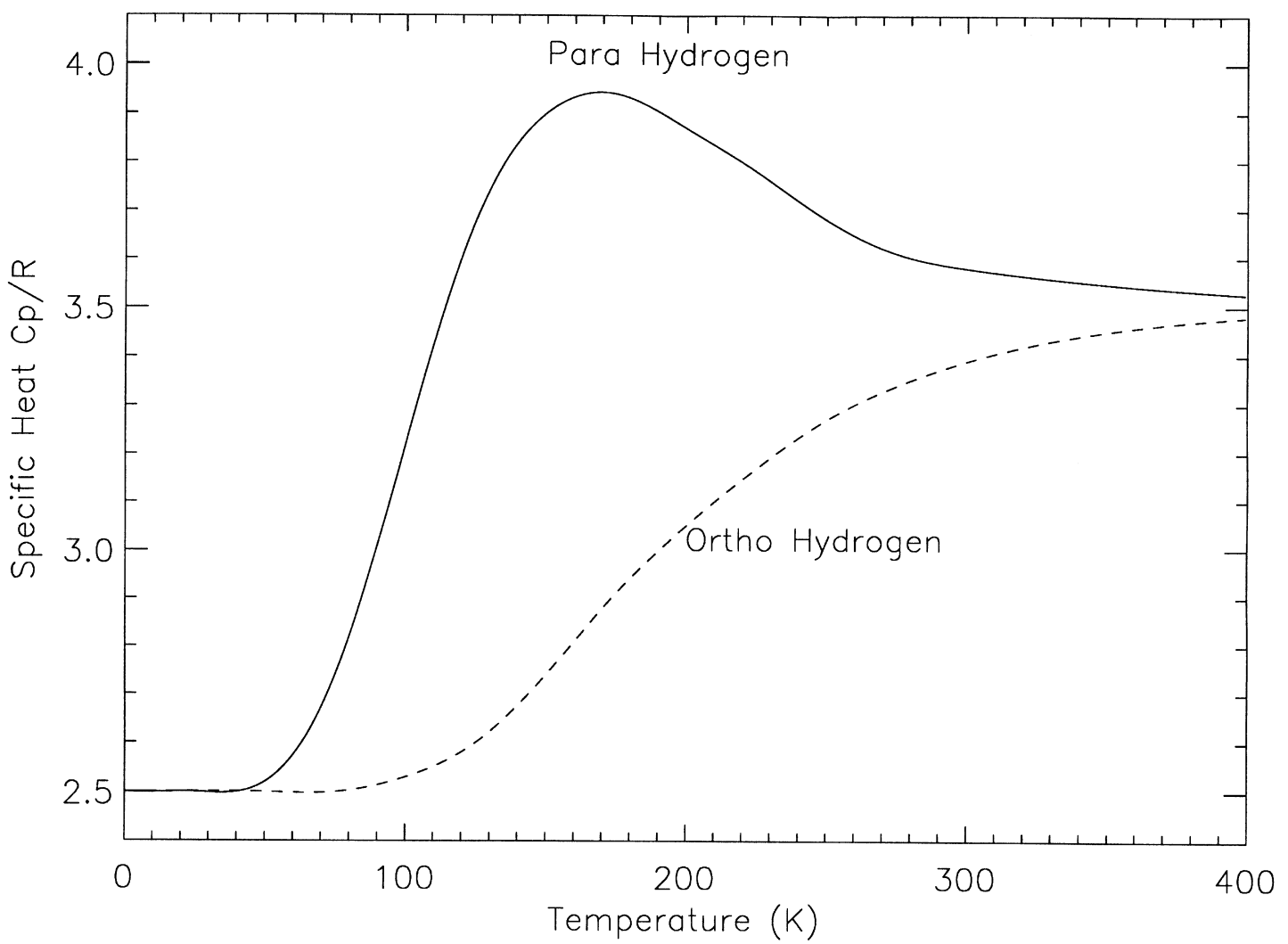

Fig. 1. The specific heats at constant pressure $\left(c_{p}\right)$ of para and ortho hydrogen, also as a function of temperature.

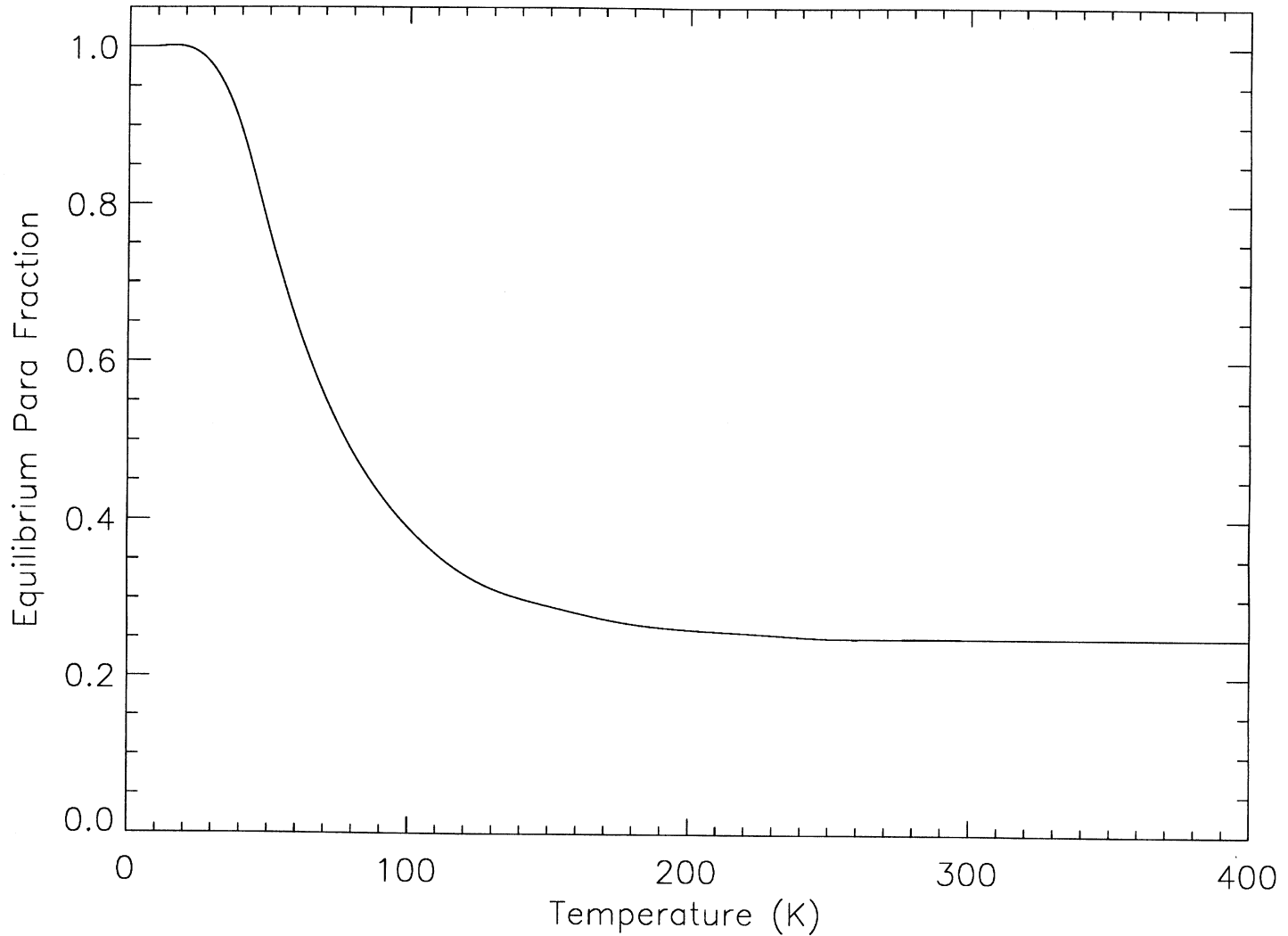

Fig. 2. The equilibrium para hydrogen fraction as a function of temperature (e.g., Massie and Hunten, 1982). 


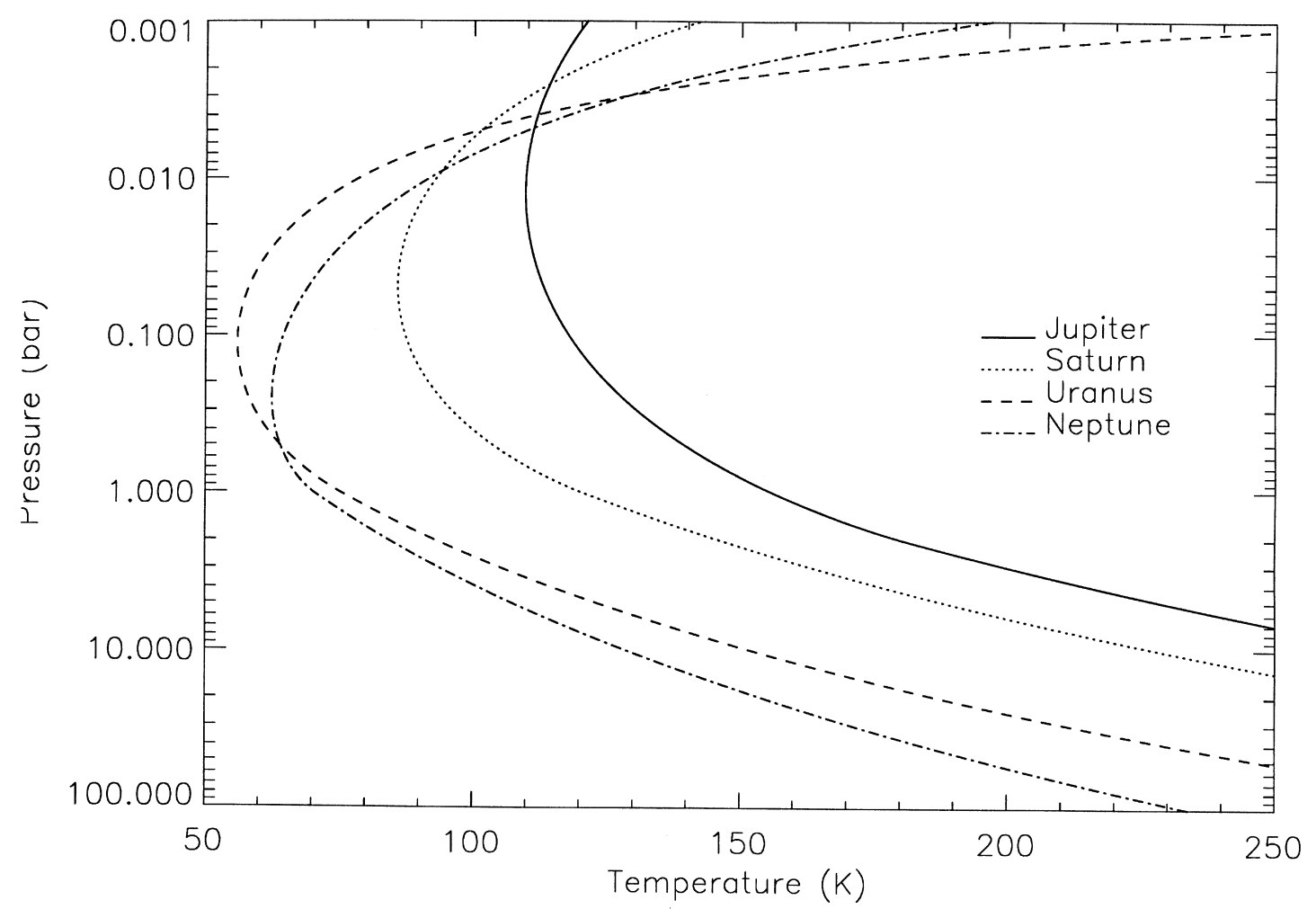

Fig. 3. Model Pressure-Temperature profiles for the four outer planets, generated as described in the text.

pressure : temperature profile is selected $\left(P_{\mathrm{ad}}, T_{\mathrm{ad}}\right)$ below which a simple lapse rate $L$ describes the profile.

Above $\left(P_{\text {ad }}, T_{\text {ad }}\right)$ the temperature versus altitude is describe by a polynomial in altitude $(z, \mathrm{~km}), T(z)=T_{\mathrm{a}}$ ${ }_{\mathrm{d}}-L z+k_{1} z^{1.5}+k_{2} z^{2}$, with $k_{1}$ and $k_{2}$ tuned so that the resultant $P / T$ profile agrees with those in the references above. The profiles are indicated in Fig. 3, and the parameters used are listed in Table 1.

The condensible species is assumed to have some fixed abundance at depth; this mixing ratio is held constant up through lower and lower pressures until the species becomes saturated (I use the saturation vapor pressure relations from Reynolds (1979)) and is then held to the

Table 1

Parameters used to generate model atmospheres

\begin{tabular}{lcccc}
\hline Parameter & Jupiter & Saturn & Uranus & Neptune \\
\hline Gravity $\left(\mathrm{ms}^{-2}\right)$ & 24.9 & 10.4 & 10.4 & 13.8 \\
$M$ & 2.3 & 2.13 & 2.3 & 2.8 \\
Lapse $\left(\mathrm{Kkm}^{-1}\right)$ & 1.8 & 0.74 & 0.87 & 1.2 \\
$P_{\text {ad }}($ bar $)$ & 2.0 & 1.0 & 1.0 & 1.0 \\
$T_{\text {ad }}(\mathrm{K})$ & 180 & 120 & 75 & 70 \\
$k_{1}\left(\mathrm{Kkm}^{-1.5}\right)$ & 0.06 & 0.02 & 0.013 & 0.18 \\
$k_{2}\left(\mathrm{Kkm}^{-2}\right)$ & $5 \mathrm{E}-3$ & $2 \mathrm{E}-3$ & $8 \mathrm{E}-3$ & $9 \mathrm{E}-4$ \\
$X \mathrm{He}^{-3}$ & 0.136 & 0.06 & 0.15 & 0.19 \\
Condensible & $\mathrm{H}_{2} \mathrm{O}$ & $\mathrm{NH}$ & $\mathrm{CH}_{4}$ & $\mathrm{CH}_{4}$ \\
Deep abundance & $4 \mathrm{E}-4$ & $2 \mathrm{E}-4$ & 0.02 & 0.025 \\
\hline
\end{tabular}

saturation curve until the minimum temperature (cold trap) is reached, and then held constant at that value. While the profiles thus generated (Fig. 4) do not take into account e.g. meteoric delivery or photochemical destruction at high altitude, they are adequate for the present purpose.

If there were no vertical transport in the atmosphere, the ortho: para hydrogen ratio would be expected to follow the temperature dependence in Fig. 2 (from Massie and Hunten, 1982). This yields the equilibrium profiles in Fig. 5. On the other hand, if gas has recently upwelled from the warm interior (where the equilibrium para fraction is 0.25 ) the 'Normal' profile (the thick line in Fig. 5) would result. In reality, the profiles will lie between these curves, depending how vigorous the vertical motions are, and how efficient aerosols are at catalysing rapid reequilibration. Chamberlain and Hunten (1987) suggest typical timescales of weeks to years for equilibration to occur, with the timescale determined mainly by the presence of paramagnetic catalysts. In a laboratory or industrial setting, the walls of the reaction vessel are the catalyst: in a planetary atmosphere, aerosols are the most likely candidate (molecular oxygen, being paramagnetic, can also accelerate equilibration, but is not abundant in the highly reducing outer planet atmospheres). These aspects are explored in some detail in Massie and Hunten (1982).

Using these model atmospheres, I compute the speed of sound for two profiles of the ortho: para ratio: one 


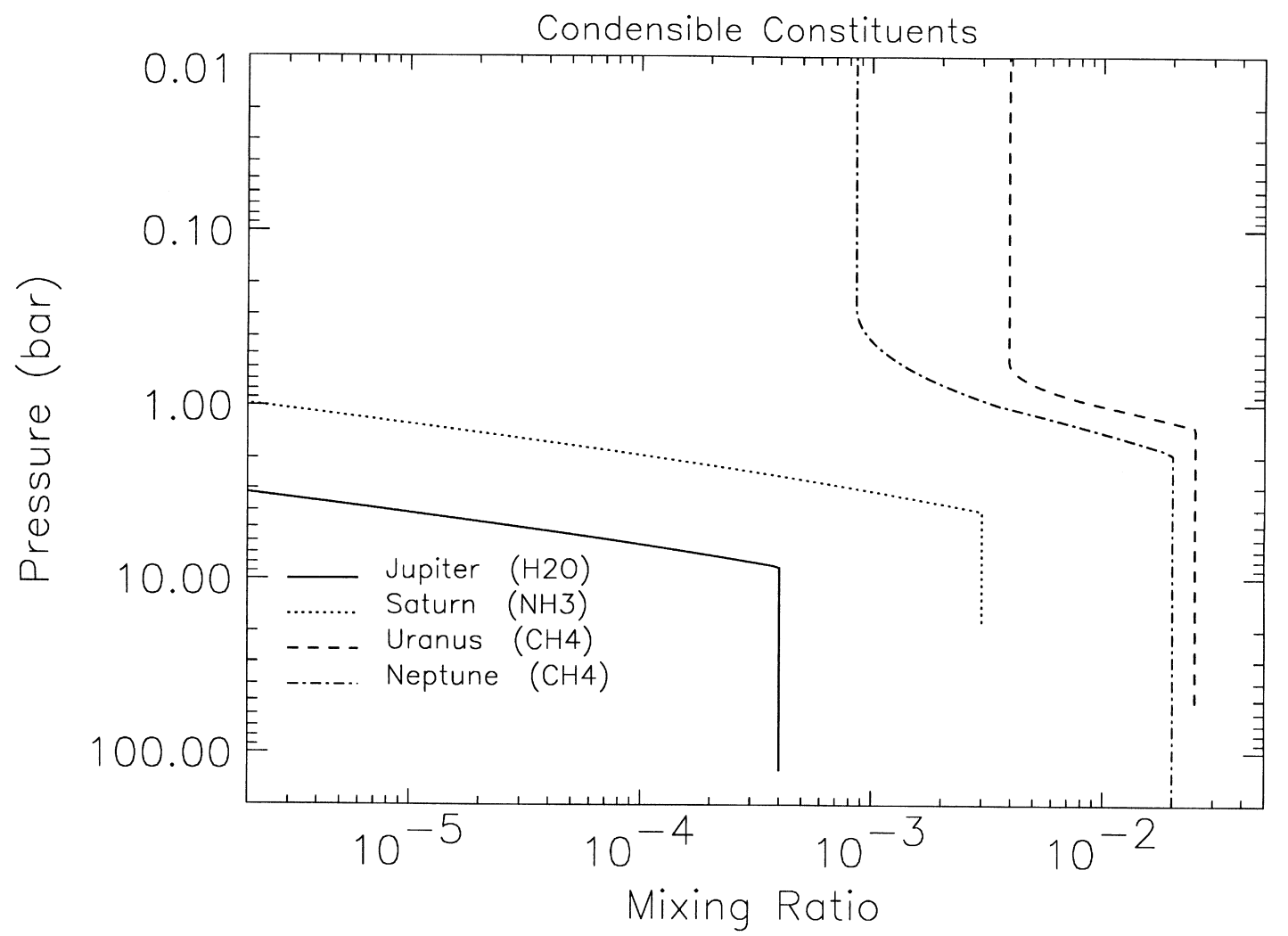

Fig. 4. Mixing ratio profiles of the condensible species. In the Jovian atmosphere the condensible (water) is too scarce here to have an appreciable effect. In Saturn's case the effect is marginal, while for both Uranus and Neptune the condensible is present at a few percent. The mixing ratios fall off at the coldtrap of each planet.

according to the equilibrium profiles in Fig. 5, and the other with a fixed normal value of 3 (para fraction $=0.25$ ); for each of these cases the condensible species is held to the profiles in Fig. 4, or at a constant deep abundance.

For convenience, $P(z)$ is computed assuming hydrostatic equilibrium using the 'deep' composition throughout; the role of the condensible in affecting the $P / T$ profile through altitude-dependent relative molecular mass is neglected in this study. Furthermore, the effects of noble gasses and non-condensing constituents (e.g., the $\sim 2 \%$ of methane in the Saturnian atmosphere) is ignored - in principle therefore the absolute values of sound speed shown in Figs $6-10$ could be offset by several percent from their true values, but the shapes are the subject of interest here. Similarly, although such effects may be significant, the influence of ortho:para hydrogen conversion on the lapse rate is ignored. For simplicity, the ratio of specific heats for the condensible constituent is assumed to be constant with temperature, and a value of 1.3 was assumed for all condensible species.

\section{Results and required accuracies}

The sound speeds for the four atmospheres are shown in Fig. 6; as might be expected the shape of the curves follow the temperature profiles fairly closely. Figures 7 10 zoom in on areas of particular interest, where the effects of condensation and ortho: para conversion are manifest in the sound profiles: each case is discussed separately below.

A straightforward error analysis indicates that uncertainties on the speed of sound propagate quadratically into uncertainty in $M$ and $\gamma$. Thus to detect the few $\%$ change in specific heat due to ortho-para conversion in the Jovian atmosphere requires that the sound speed to be measured to a fraction of a percent.

Also, in this same example, uncertainty in composition (and hence assumed $M$ ) is commensurate with the resultant uncertainty in $\gamma$. For Jupiter, this is less of a concern, in that the hydrogen: helium ratio is well-determined (Von Zahn and Hunten, 1996) and all other constituents are present at levels below $0.5 \%$ (Niemann et al., 1996). In the other planets, however, tropospheric mixing ratios of methane and ammonia are rather higher, yet fall across cold-traps where clouds form. In these cases, the speed of sound curves show a sharp change in slope.

The speed of sound measurement for Jupiter would have to be better than $0.2 \%$ accurate to detect either the onset below some altitude of a water abundance equal to that measured by Galileo (although wetter regions on Jupiter may be rather easier to detect), or the effect of 


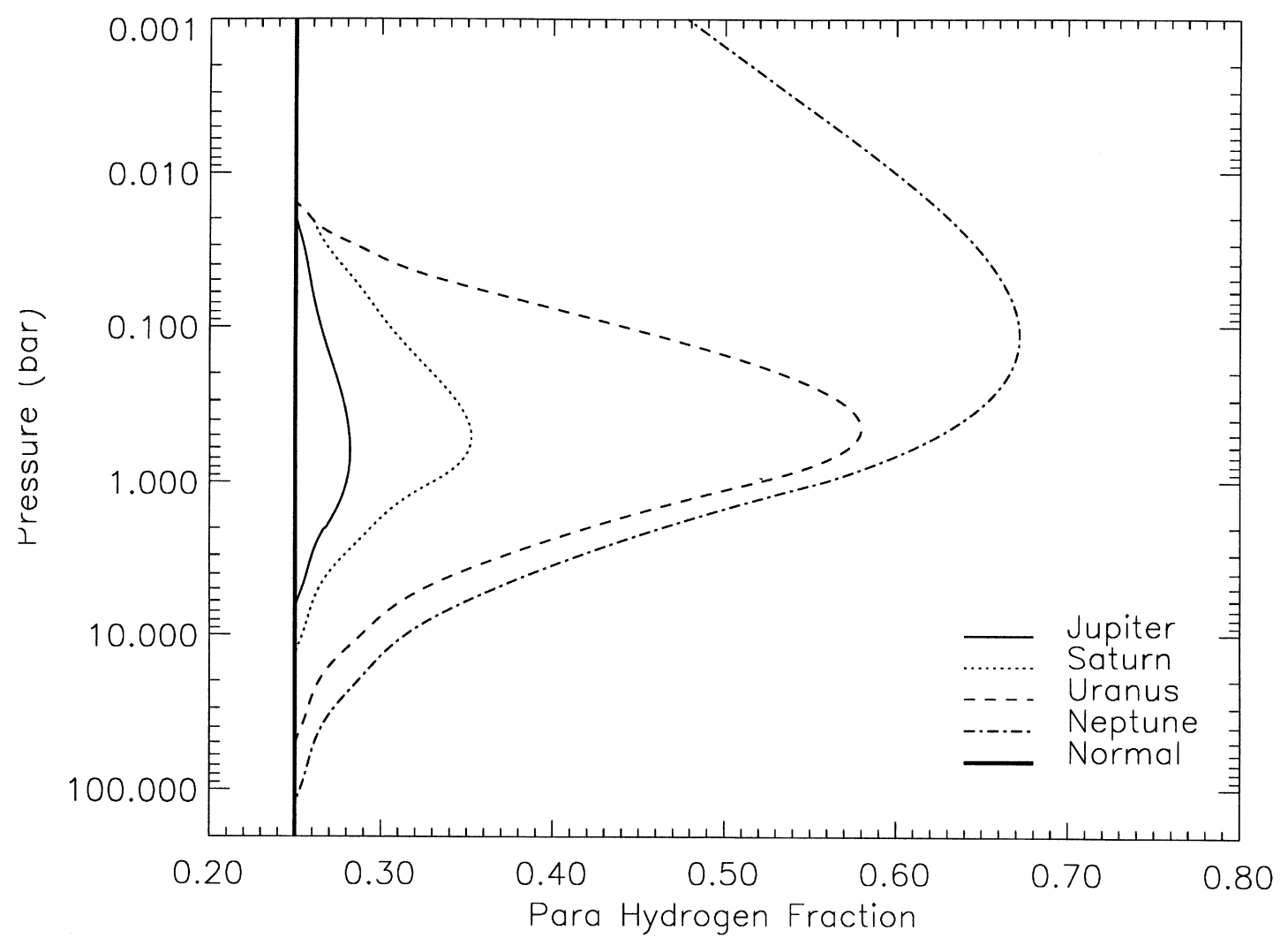

Fig. 5. Para hydrogen fraction profiles. The thin lines correspond to local equilibrium (the temperature profile is operated on by the function in Fig. 1). The thick line indicates the 'Normal' (high temperature equilibrium) value, that might be expected if upwelling from the interior were rapid.

para hydrogen (Fig. 7). For a useful measurement (rather than a mere detection), an accuracy of perhaps 10 times better is needed, so Jupiter seems per se a marginal prospect for this technique.

For Saturn, again the condensible detection is somewhat marginal owing to the relatively low abundance. The effect of ortho : para conversion is about $0.5 \%$, so a sensor with a resolution of $0.5-1 \mathrm{~ms}^{-1}$, or 1 part in 1000 , could make a useful contribution (Fig. 8).

The technique really comes into its own for the two outermost giant planets, which have both higher condensible abundance, and lower temperatures (hence higher equilibrium para hydrogen fractions). Detection of layers where the equilibrium para hydrogen fraction is attained above layers where it is closer to the hightemperature value might indicate aerosol or cloud layers. Models might be able to reconstruct the vertical mixing rate from the observed para hydrogen ratio, if the recombination rate can be adequately constrained.

The musically-inclined reader might note that the change in sound speed due to the change in methane abundance across the Neptune cold trap would change the note of a whistle (say, an F at $350 \mathrm{~Hz}$ ) to an F-sharp (F\#, at $370 \mathrm{~Hz}$ ).

\section{Instrumental considerations}

The speed of sound measurement advocated by Hanel and Strange (1966) used the phase difference between a number of transducers in a temperature-controlled spiral duct. While suitable for a fixed in-situ measurement with plenty of time to equilibrate, such a technique may be difficult for a probe plummeting through an atmosphere with strong vertical variation in temperature: the heaters in the duct would have to compensate for a rapidlychanging gas temperature: naturally, most information on the external gas temperature is lost. However, if the temperature-regulation challenges could be overcome, phase difference measurements offer intrinsically high accuracy.

The time-of-flight measurement may be a viable alternative, and is the technique implemented on the Huygens SSP instrument (Zarnecki et al., 1997). Time is one of the simplest things to measure in an instrument; the measurement uncertainty is therefore dominated by the uncertainty in the pathlength. This might be ameliorated either by stiff construction and/or measuring in real time (perhaps by optical means) the pathlength. As on SSP, the propagation time in both directions should be measured 


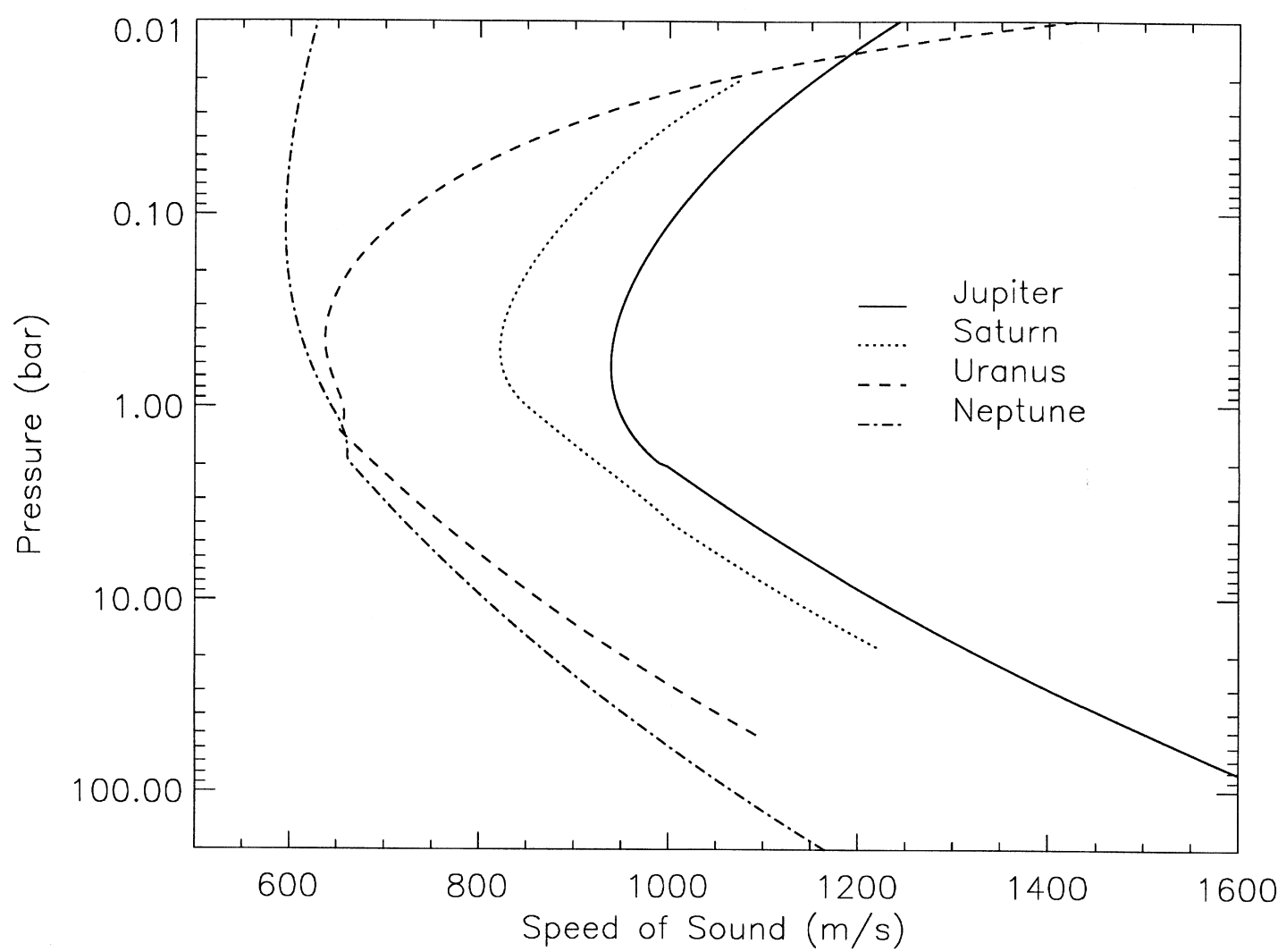

Fig. 6. Speed of sound profile for the four planets, assuming equilibrium para hydrogen fraction, and variable condensible abundance. The kinks in the curves are due to the sharp change in mixing ratio of condensible species.

to eliminate the effects of airflow (indeed, the difference in sound speeds along and against the vehicle's direction of descent might place a useful independent constraint on the probe descent velocity).

Resonant-cavity methods (i.e., whistles), alluded to frivolously in the previous section, might in fact make a viable sound speed sensor. Measuring the frequency of a whistle excited by the draught of the probe's descent would again be straightforward. Turbulence may be an accuracy-limiting factor for all techniques, and adequate gas flow needs to be ensured so that the instrument tracks external composition variations adequately.

An experimental programme would probably be required to determine the most accurate sensors, and their range of operation. The high pressure limit is probably driven by the probe itself; the temperatures beyond which transducers cannot operate are very high (cf their use in exhaust gas measurement). Piezoelectric transducers would be ultimately limited by their Curie temperature (where they become depolarized, typically a few hundred degrees Centigrade), although this is not likely to be a significant limitation except for very deep (100 bar + ) probes.

The low pressure limit will be determined by signal : noise considerations: at lower pressure the coupling of the atmosphere to the transducer becomes poorer and poorer. The frequency of operation might be chosen to maximize the signal to noise (higher frequencies are attenuated more strongly - probably not an important issue for such short pathlengths-while lower frequencies suffer more aeroacoustic noise).

The speed of sound measurement in itself can provide useful constraints on transport and condensation. It is likely to be more valuable yet combined with other measurements: condensation might be indicated via the lapse rate, and optical extinction measured by a radiometer, or by SODAR, detecting backscatter from raindrops. Frequency-dependent backscatter would allow the dropsize distribution to be constrained.

All the interpretations of sound speed described in this paper require that the gas temperature be known accurately.

\section{Conclusions}

The models outlined in this paper, while neither rigorous nor complete, describe the vertical variation of sound speed within each atmosphere. It is found that variations in sound speed are a very accessible diagnostic of the relative molecular mass and ortho : para hydrogen ratio in the atmospheres of Uranus and Neptune, but 

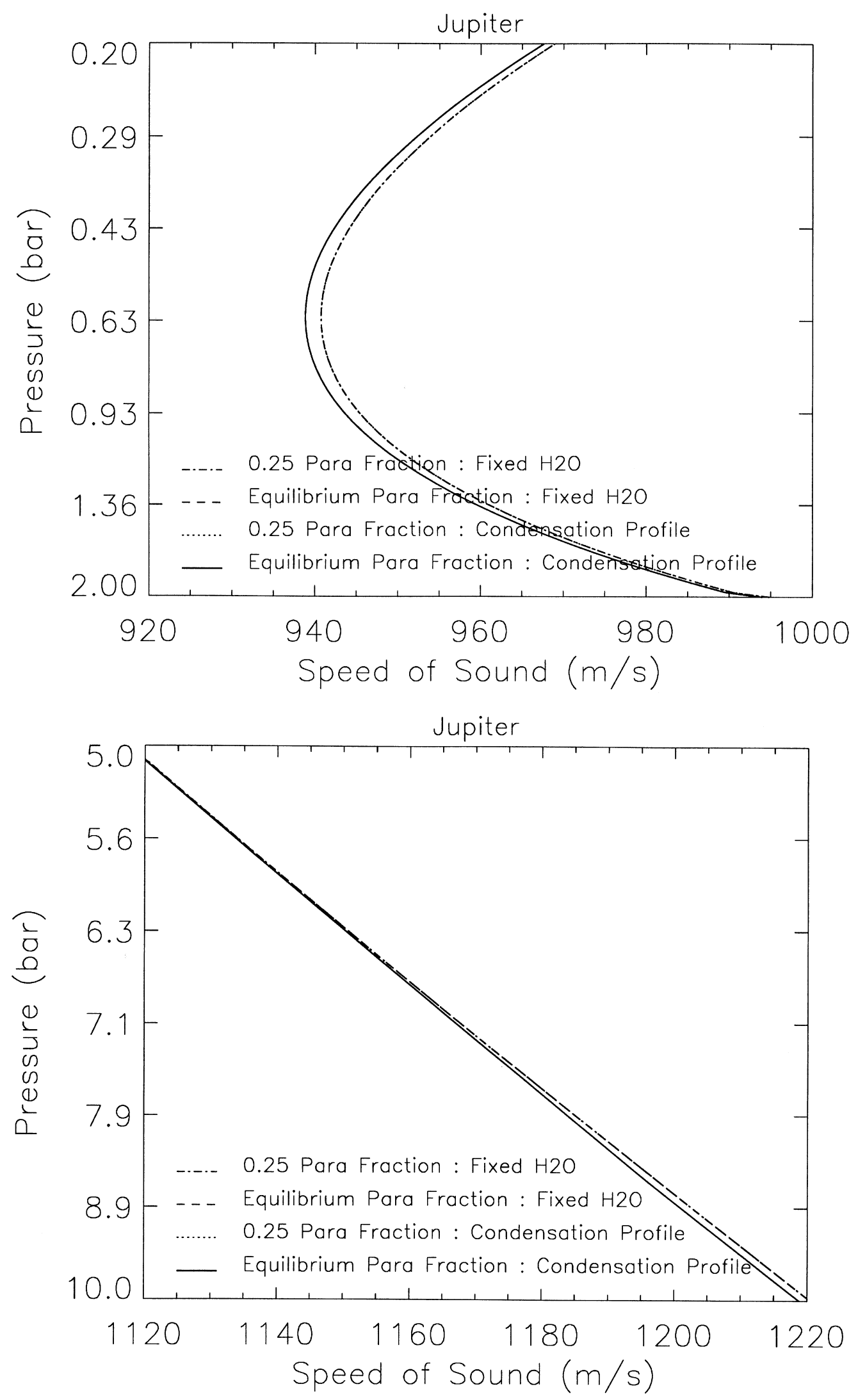

Fig. 7(a). Detail of the speed of sound profile for Jupiter, over the $0.2-2$ bar range. The curves for different cases of condensible mixing ratio cannot be discriminated (above the cold trap the water abundance is too small), but the non-equilibrium ortho: para ratio causes a difference of $\sim 2 \mathrm{~ms}^{-1}$ from the equilibrated cases; (b) detail of the speed of sound profile in the 5-10 bar range. The deep abundance of water makes a $\sim 2 \mathrm{~ms}^{-1}$ difference in sound speed. 

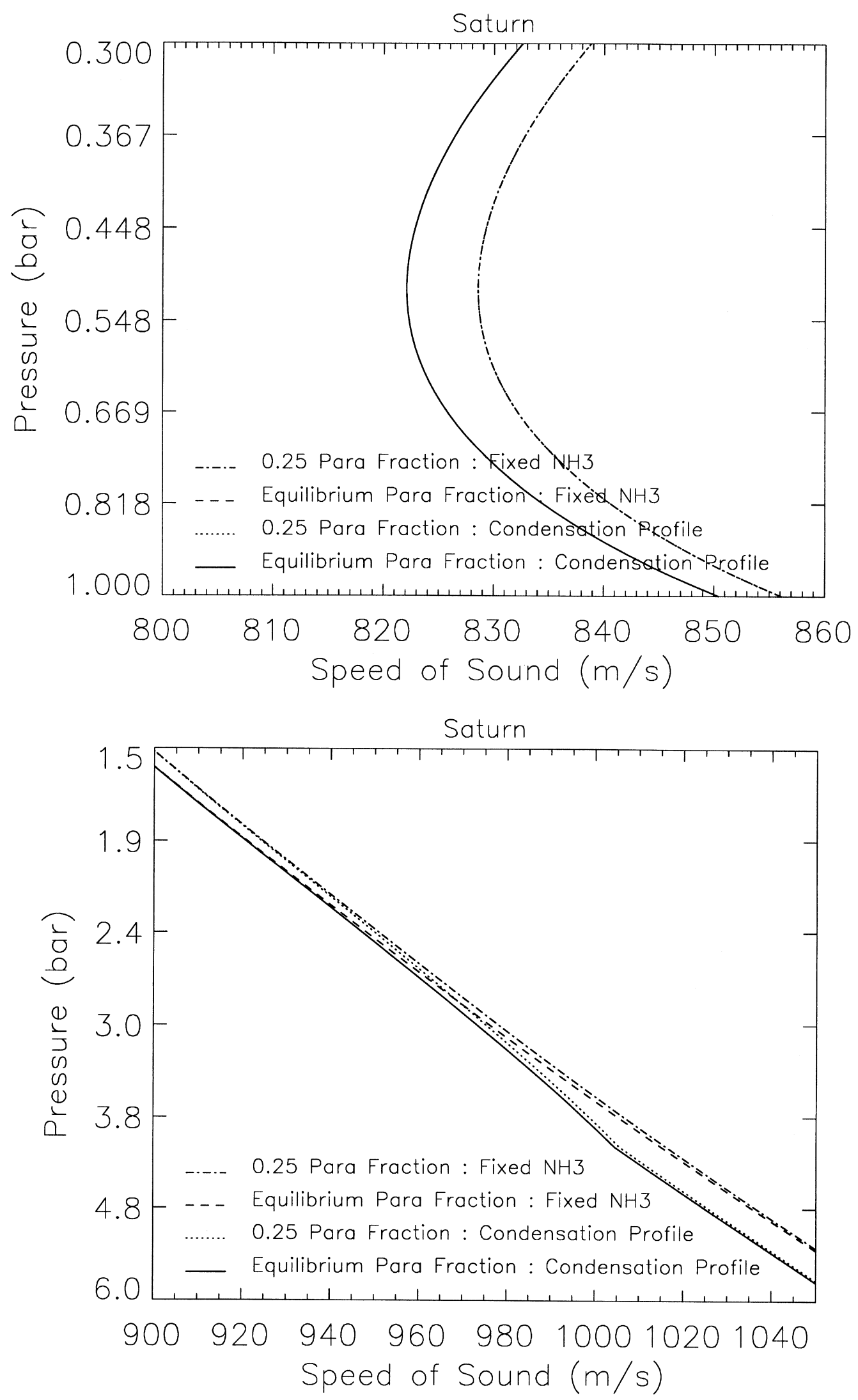

Fig. 8(a). Detail of the speed of sound profile for Saturn, over the $0.2-1$ bar range. The curves for different cases of condensible mixing ratio cannot be discriminated (above the cold trap the ammonia abundance is too small), but the non-equilibrium ortho: para ratio causes a difference of $\sim 5$ $\mathrm{ms}^{-1}$ from the equilibrated cases; (b) detail of the speed of sound profile in the 5-10 bar range. The deep abundance of ammonia makes a $\sim 2 \mathrm{~ms}{ }^{-1}$ difference in sound speed. 


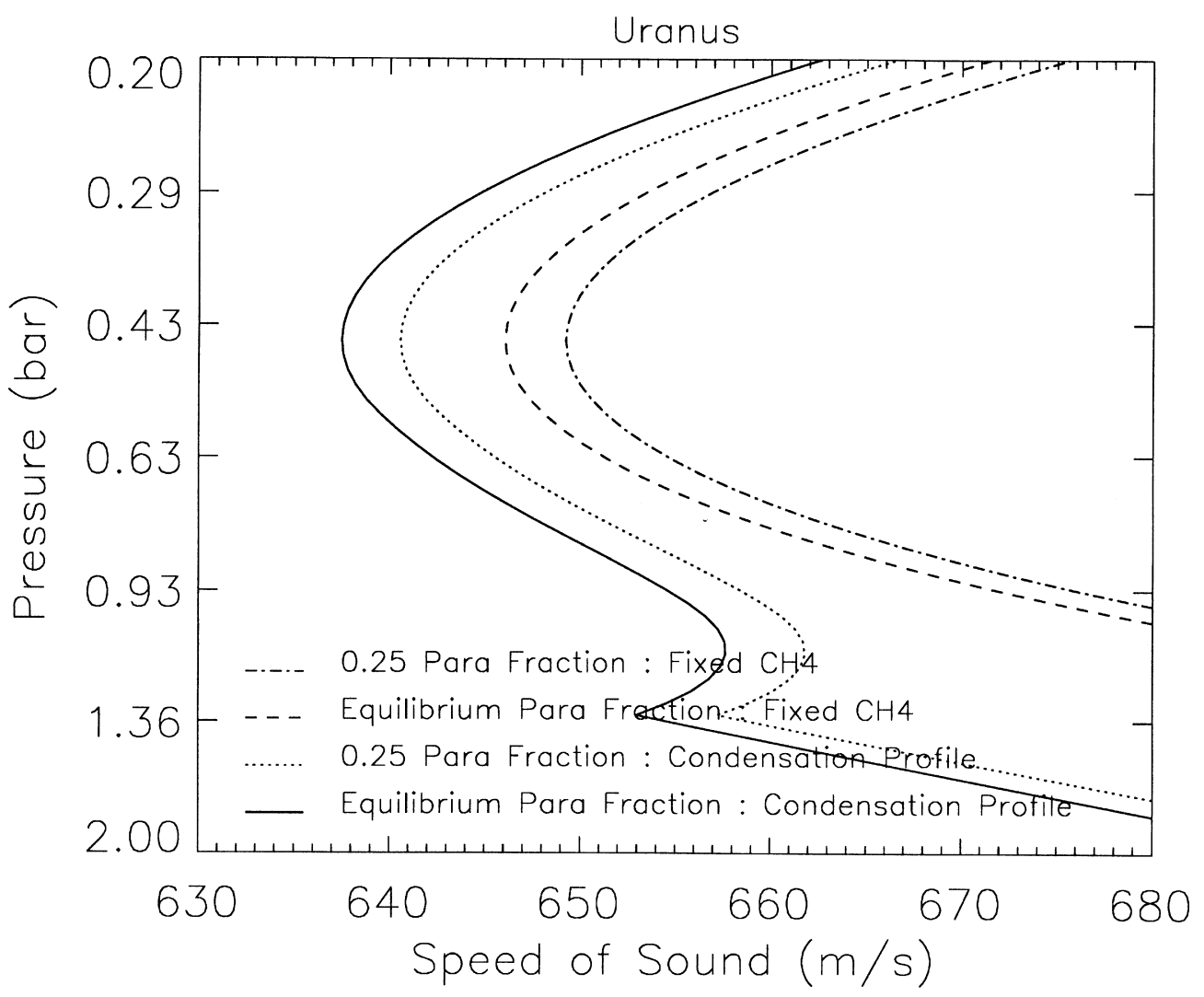

Fig. 9. Detail of the speed of sound profile for Uranus, over the 0.2-2 bar range. The very low temperatures allow a large para hydrogen fraction, and even at the cold trap the methane abundance is significant, so all the curves are separate. The influence of condensation on the sound speed (via $\mathrm{RMM}$ ) is striking.

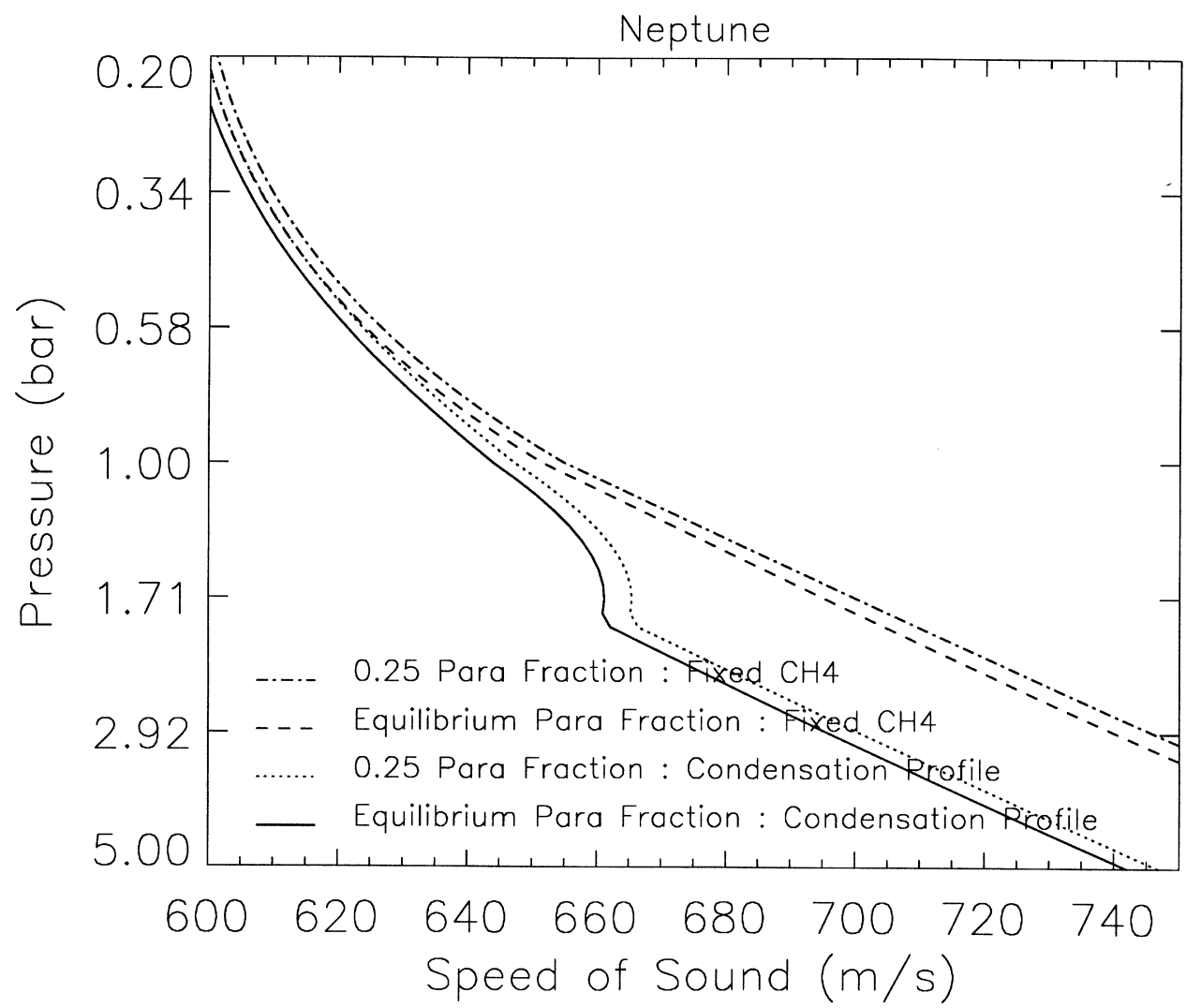

Fig. 10. Detail of the speed of sound profile for Neptune, over the 0.1-2 bar range. The methane mixing ratio profile again makes a difference of some $40 \mathrm{~ms}^{-1}$ below 2 bar. Above about 0.8 bar, the effects of the methane mixing ratio and the ortho: para ratio are approximately equal, so the dashed and dotted curves merge. 
challenge available instrumental accuracies for Jupiter. Saturn is a marginal prospect-with modest technical effort a suitable instrument could be constructed.

Given the modest mass, power and data requirements of a speed of sound sensor, it may be a valuable and costeffective complement to other instrumentation on future outer planet probes.

\section{References}

Chamberlain, J.W., Hunten, D.M., 1987. Theory of Planetary Atmospheres, 2nd ed. Academic Press, San Diego.

Conrath, B.J., Gierasch, P.J., 1984. Global variation of the para hydrogen fraction in Jupiter's atmosphere and implications for dynamics on the outer planets. Icarus 57, 184-204.

Conrath, B.J., Pearl, J.C., Appleby, J.F., Lindal, G.F., Orton, G.S., Bezard, B., 1991. Thermal structure and energy balance of Uranus. In: Bergstrahl, J.T., Miner, E.D., Matthews, M.S. (Eds), Uranus. University of Arizona Press, Tucson. pp. 204-252.

Estrada-Alexanders, A.F., Trusler, J.P.M., Zarari, M.P., 1995. Determination of thermodynamic properties from the speed of sound International Journal of Thermophysics 16, 663-673.

Fegley, B. Jr., Gautier, D., Owen, T., Prinn, R.G., Spectroscopy and chemistry of the atmosphere of Uranus. In: Bergstrahl, J.T., Miner, E.D., Matthews, M.S., (Eds), Uranus. University of Arizona Press, Tucson. pp. 147-203.

Fulchignoni, M., Angrilli, F. Bianchini, G., Bar-Nun, A., Barucci, M., Borucki, W., Coradini, M., Coustenis, A., Ferri, F., Grard, R. J., Hamelin, M., Harri, A.M., Leppelmeier, G.W., JLopez-Moreno J., McDonnell, J.A.M., McKay, C., Neubauer, F.M., Pedersen, A., Picardi, G., Pirronello, V., Pirjola, R., Rodrigo, R., Schwingenschuh, C., Seiff, A., Svedhem, H., Thrane, E., Vanzani, V., Visconti, G., Zarnecki, J., 1997. The Huygens atmospheric structure instrument (HASI). In: Wilson, A., (Ed.), Huygens: Science, Payload and Mission. ESA SP-1177, European Space Agency, Noordwijk, The Netherlands. pp. 163-176.

Gautier, D., Conrath, B.J., Owen, T., De Pater, I., Atreya, S.K, 1995. The troposphere of Neptune. In: Cruikshank, D.P. (Ed.). Neptune and Triton. University of Arizona Press, Tucson. pp. 547-612.

Hanel, R.A., Strange, M.G., 1966. Acoustic experiment to determine the composition of an unknown planetary atmosphere. Journal of the Acoustical Society of America 40, 896-905.

Ingersoll, A.P., Beebe, R.F., Conrath, B.J., Hunt, G.E., 1984. Structure and dynamics of Saturn's atmosphere. In: Gehrels, T., Matthews, M.S., (Eds), Saturn. University of Arizona Press, Tucson. pp. 195238.

Kleppe, J.A., 1996. High temperature acoustic pyrometry. Sensors 13, $17-22$.

Ksanfomality, L.V., Scarf, F.L., Taylor, W.L., 1983a. The electrical activity of the atmosphere of Venus. In: Hunten, D.M., et al., (Eds), Venus. University of Arizona.

Ksanfomality, L.V., Goroshkova, N.V., Khondryev, V.K., 1983b. Wind velocity near the surface of Venus form acoustic measurements.
Cosmic Research 21, 161-167 (translated from Kosmicheskii Issledovania 21, 218-224.

Lewis, J.S., 1995. Physics and Chemistry of the Solar System. Academic Press, San Diego.

Lindal, G. F., Wood, G. E., Hotz, H. B., Sweetnam, D. N., Eshleman, V. R. and Tyler, G. L., 1983. The atmosphere of Titan - an analysis of the Voyager 1 radio occulation measurements. Icarus 53, 348363.

Massie, S.T., Hunten, D.M., 1982. Conversion of para and ortho hydrogen in the Jovian planets. Icarus 49, 213-226.

Neff, W.D., Coulter, R.L., 1986. Acoustic remote sensing. In: Lenschow, D.H., (Ed.), Probing the Atmospheric Boundary Layer. American Meteorological Society, Boston, pp. 201-238.

Niemann, H.B., Atreya, S.K., Carignan, G.R., Donahue, T.M., Haberman, J.A., Harpold, D.N., Hartle, R.E., Hunten, D.M., Kasprzak, W.T., Mahaffy, P.R., Owen, T.C., Spenser, N.W., Way, S.H., 1996. The Galileo probe mass spectrometer: Composition of Jupiter's atmosphere, Science 272, 846-849.

Prinn, R.G., Larson, H.P., Caldwell, J.J., Gautier, D. 1984. Composition and chemistry of Saturn's atmosphere. In: Gehrels, T., Matthews, M.S., (Eds), Saturn. University of Arizona Press, Tucson. pp. $88-149$.

Reynolds, W.C., Thermodynamic Properties in SI. Stanford University, 1979.

Rogers, G.F.C., Mayhew, Y.C. Engineering Thermodynamics: Work and Heat Transfer, Longman, London, 1967.

Seiff, A., Kirk, D.B., Knight, T.C.D., Mihalov, J.D., Blanchard, R.C., Young, R.E., Schubert, G., von Zahn, U., Lehmacher, G., Milos, F.S., Wang, J., 1996. Structure of the atmosphere of Jupiter: Galileo Probe measurements. Science 272, 849-851.

Smith, M.D., Gierasch, P.J., 1995. Convection in the outer planet atmospheres including orth-para hydrogen conversion. Icarus 116, 159-179.

Spiesberger, J.L., Tappert, F.D., 1996. Kaneohe acoustic thermometer further validated with rays over $3700 \mathrm{~km}$ and the demise of the idea of axially trapped energy. Journal of the Acoustical Society of America 99, 173-184

Tyndall, J. Sound. Appleton, New York, 1896.

Von Zahn, U., Hunten, D.M., 1996. The helium mass fraction in Jupiter's atmosphere. Science 272, 849-851.

Weill, A., Lehmann, H.-R., 1990. Twenty years of acoustic soundinga review and some applications. Zeitschrift fur Meteorologie 40, 241250.

Yelle, R.V., Strobell, D.F., Lellouch, E., Gautier, D., 1997. Engineering models for Titan's Atmosphere. In: Wilson, A., (Ed.), Huygens: Science, Payload and Mission, ESA SP-1177, European Space Agency, Noordwijk, The Netherlands. pp. 253-256.

Zarnecki, J.C., Banaszkiewicz, M., Bannister, M., Boynton, W.V., Challenor, P., Clark, B., Daniell, P.M., Delderfield, J., English, M.A., Fulchignoni, M., Garry, J.R.C., Geake, J.E., Green, S.F., Hathi, B., Jaroslawski, S., Leese, M.R., Lorenz, R.D., McDonnell, J.A.M., Merryweather-Clarke, N., Mill, C.S., Miller, R.J., Newton, G., Parker, D.J., Svedhem, L.H., Turner, R.F., Wright, M.J., The surface science package, In: Wilson, A. (Ed.), Huygens: Science, Payload and Mission. ESA Special Publication SP-1177, pp. 177195. 